\title{
Design Analysis Method for Multidisciplinary Complex Product using SysML
}

\author{
Jihong Liu ${ }^{1, *}$, Shude Wang ${ }^{1}$, and Chao Fu ${ }^{1}$ \\ ${ }^{1}$ School of Mechanical Engineering and Automation, Beihang University, 100191 Beijing, China
}

\begin{abstract}
In the design of multidisciplinary complex products, model-based systems engineering methods are widely used. However, the methodologies only contain only modeling order and simple analysis steps, and lack integrated design analysis methods supporting the whole process. In order to solve the problem, a conceptual design analysis method with integrating modern design methods has been proposed. First, based on the requirement analysis of the quantization matrix, the user's needs are quantitatively evaluated and translated to system requirements. Then, by the function decomposition of the function knowledge base, the total function is semi-automatically decomposed into the predefined atomic function. The function is matched into predefined structure through the behaviour layer using function-structure mapping based on the interface matching. Finally based on design structure matrix (DSM), the structure reorganization is completed. The process of analysis is implemented with SysML, and illustrated through an aircraft air conditioning system for the system validation.
\end{abstract}

\section{Introduction}

In the process of complex product design, system engineering is a kind of development methods which covers a wide range of applications across from system requirements analysis, function decomposition to physical construction, and so on. The purpose is to ensure that the user's needs are satisfied throughout the whole life cycle of the system and that the efficiency benefits are maximized [1]. With the increase of the complexity of the product, the document-based system engineering is difficult to meet the current $R \& D$ requirements, and the model-based system engineering (MBSE) becomes the best choice in the context of integrating the multidisciplinary and multidisciplinary background. The core idea is to model the development of all the information related to the system design from the beginning of the system research and development, and as a central system model throughout the product system life cycle.

However, the existing MBSE methodology only contains the modeling process of each stage, experience-based analysis process, and short of rigorous analysis theory and analysis methods [2]. The existing analysis theory is difficult to combine the characteristics of SysML model, and can't be good for SysML Expression. It is also difficult to support the whole process of model establishment. In this paper, by combining the conceptual design analysis theory and the expression characteristics of SysML model, a design analysis method is proposed to support the conceptual design stage of complex product in the multi-disciplinary field with the requirement analysis, function decomposition and function modeling, function structure mapping and evaluation, and structure reorganization. The whole process of the analysis method is aimed at providing designers with an effective analysis of ideas and theoretical support to reduce the bad design and improve design efficiency.

The paper is organized as follows: The second part of the article briefly introduces the relevant theoretical basis, the third part describes the specific implementation process of the method, and the forth part uses the aircraft air conditioning system as an example to verify the effectiveness of the method. The last part is the article summary.

\section{Theoretical}

\subsection{MBSE methodology}

The contribution of MBSE to short the development cycle and reduce the cost, not only rely on the ability to describe a strong system modeling language, but also need the support of scientific methodology. From a broad sense of conceptual perspective, MBSE methodology is a collection of processes, languages, and tools that support the full range of product development under "modeled" trends. At present, the mainstream methodologies include [3]: IBM Harmony-SE, INCOSE object-oriented system engineering method, using the "onion" model of the Vitech MBSE methodology, RUP SE methodology developed from the Software Development Unified Process Framework (RUP), Product system modeling methods based on the complex

"Corresponding author: ryukeiko@buaa.edu.cn 
state analysis. Although the modeling order and the analysis process are different in different methodologies, they do not include specific analytical methods.

\subsection{SysML}

SysML has the characteristics of object-oriented, graphical, platform-independent, and can describe, analyse, design and verify the complex systems covering software, hardware and other information, and provide visual semantics expression for system requirements, behavior, structure and parameter modeling to ensure that model-based research and development can cover through the system design to the implementation of the various stages [4]. Combined with the characteristics of system engineering, SysML has been improved based on the UML, and model the whole conceptual design phase by expressing the four types of design information (requirement, behavior, structure and parameters) of the system through nine graphs. The requirement and parameter diagram is the new diagrams in SysML.

\section{3 modern design methods}

Modern design theory can provide the theoretical basis for MBSE analysis. There are many kinds of modern product design methods.

In the combination of modern design method and MBSE, Wang [2] studied the system engineering method and MBSE model system based on axiomatic design, and combined the axiomatic design process with MBSE modeling system. But the core theory of axiomatic design can't support the whole process of conceptual design.

Yuan and Liu [7] studied the function decomposition using SysML. And Giacomo [8] preposed a method to the development of mechatronic systems based on SysML. However, those methods are not able to support the the whole process of design.

\section{Multidisciplinary complex product design analysis method}

\subsection{Requirement analysis based on quantization matrix}

Requirements analysis is an important stage in the process of product design. Quality Function Deployment (QFD) which is a widely used design method is used in the conceptual design phase to ensure that the requirements from the customer or market is accurately transferred to the product life cycle by analysing the quality house each stage of the relevant technology and measures. In this paper, through the application of QFD thinking, the user needs into a standardized system requirements description, while the use of analytical matrix, analysis and evaluation, in order to get more in line with product system requirements. The specific process is shown in Fig.1.

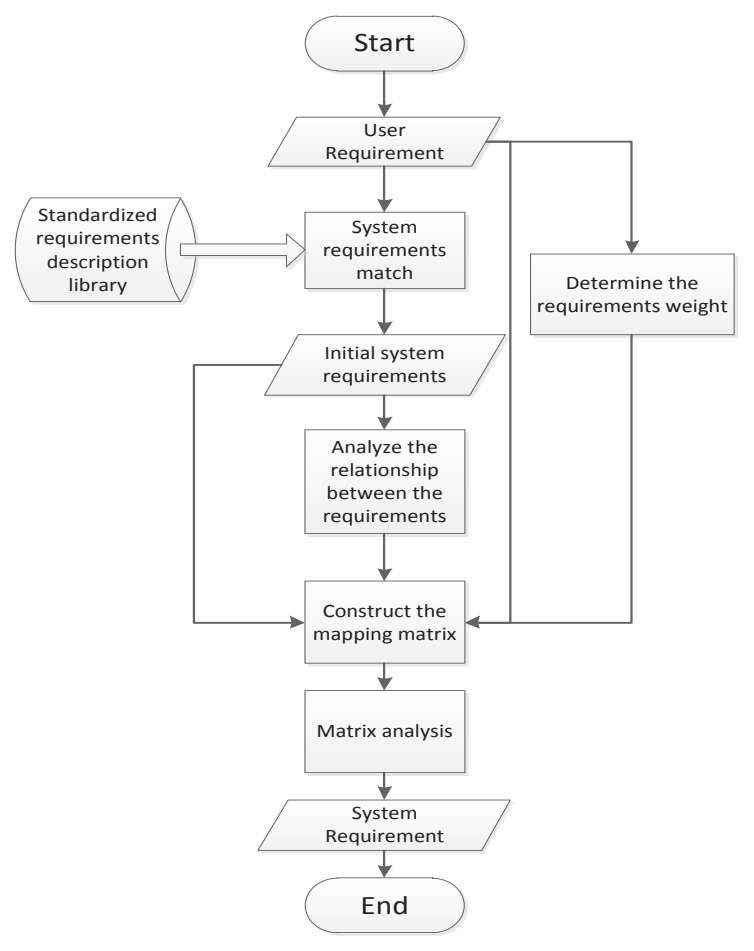

Fig.1. The process of requirement analysis

Step 1: according to the product properties, the technical staffs establish this type of product standardization requirements description library, such as air conditioning refrigeration, price and other content, to transport the non-standard requirement description provided by the customers, into the system requirements described with the standard technical terms in this domain.

Step 2: establishing the mapping matrix user needs to expand system requirements, the application of quality function of thought (QFD), the user needs to weigh mapped to the system requirements. At the same time, the description of the requirement relation is extended and the requirement relation of the system is analysed. The requirement relationship includes the inclusion, trace, derive, refine, satisfy, and verify, to quantify several relationships to determine the system requirements. The standard of quantifying is shown in the Table 1.

Table 1. The standard of quantifying

\begin{tabular}{|l|c|c|c|c|c|c|}
\hline Relationship & Contain & Trace & Derive & Refine & Satisfy & Verify \\
\hline Weight & 1.0 & 0.9 & 0.7 & 0.5 & 0.3 & 0.1 \\
\hline
\end{tabular}

Step 3, the resulting system requirements for attribute analysis, the property is defined as the functional requirements (describe the system function requirements type) and non-functional requirements (other types of needs, including price, size constraints), that is, system functions and Product constraints.

\subsection{Function decomposition based on function knowledge base}

The function modeling of the product is realized through the process of function decomposition. There are many ways to realize the function decomposition. According to 
the characteristics of the SysML, this paper proposes the function decomposition method based on the function knowledge basis. In this method, the qualitative processing reasoning (QFR) [6] theory is applied. The change type is divided into four types: type change, numerical change, value space change and derivative change. The analysis of the change type is carried out to complete the function decomposition based on flow. The specific process is shown in Fig.2.

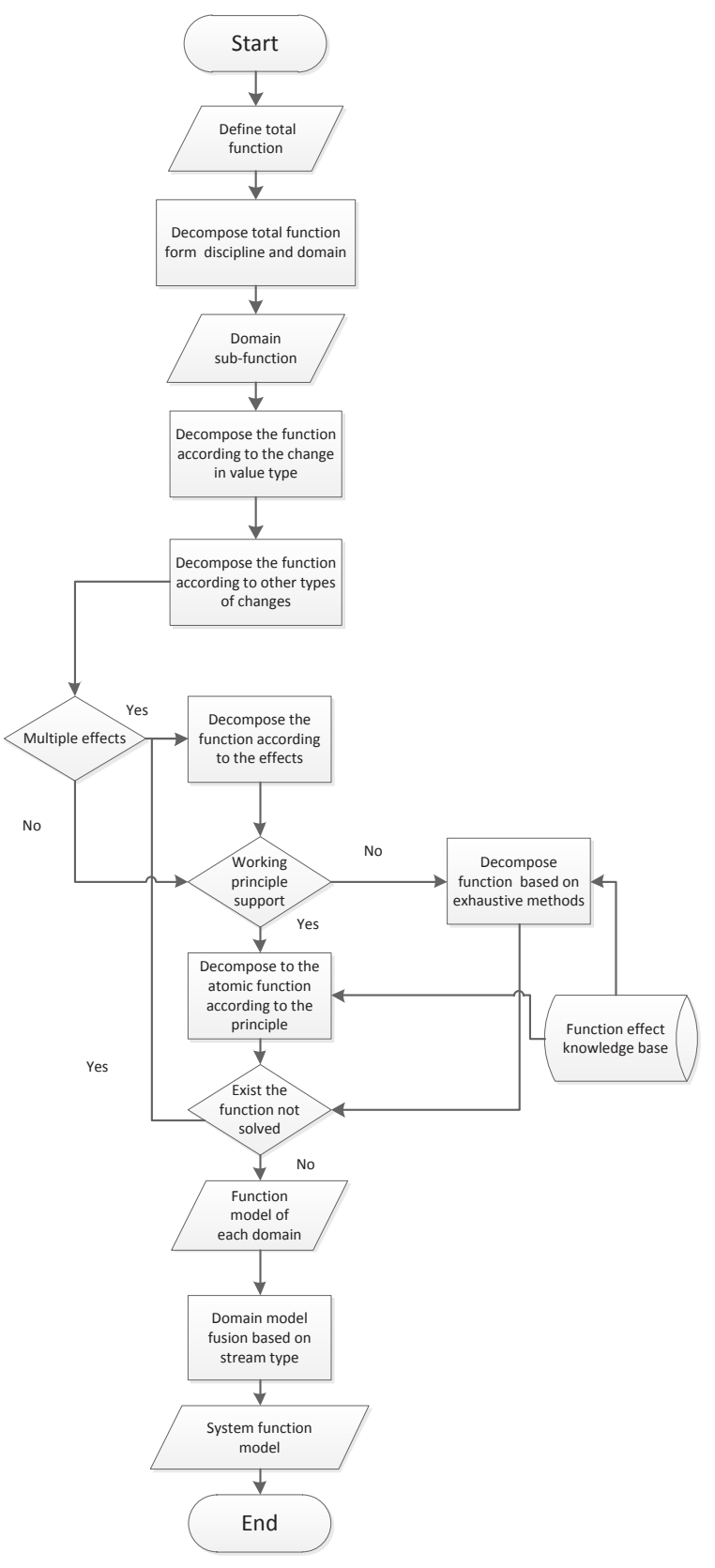

Fig. 2. The process of function decomposition

Step 1: According to the characteristics of multi-disciplinary and multidisciplinary, the overall function of the system is decomposed into the overall functions of the sub-systems according to the characteristics of the sub-systems, such as the control sub-system, the mechanical sub-system, and the total function of the subsystems defined by the input and output flow changes of the subsystems.

Step 2: Designers of different domains decompose the function of their sub-system according to the following principles: First, according to the change of the value of the stream, the change of the value type is defined as the result of the decomposition of the first layer. And then the decomposition of the sub-function on the next layer, according to the value of change, the value of space changes, derivative changes again function decomposition, the formation of the next level of function.

Step 3: Establish the function effect knowledge base, and define the atomic function (that is the function that isn't needed re-decomposition function), and the function based on the physical or chemical effects, such as the Joule effect, the effect of the input and output flow changes. The results of the previous analysis can be matched to support the effect of the change, and then decomposed into atomic functions. For the sub-functions which can't be matched the appropriate atomic function through the effect, all the atomic functions will be retrieved and matched according to the change type of input and output streams through the exhaustive method. For the function that isn't able to be deposed, expert opinion is needed to redefine the atomic function, and then need to be added to the knowledge base, so as to get a complete hierarchical function model.

\subsection{Function-structure mapping based on interface matching}

After the function modeling is completed, the function-structure mapping needs to be established. The result of the function model is mapped to the structure in order to obtain the overall structure model. The physical structure to realize the atomic function can be deduced through the function model [6], and the connections between the structure interfaces need to be established to achieve the complete system conceptual design scheme. The mapping process is divided into two categories. The first one is the function-structure direct mapping, mainly through the port matching relationship, which can provide an alternative design for the structure. The other makes use of the expected behavior as an intermediate transition layer, to realize the function-behavior-structure of the three layers model mapping. The former mainly focuses on the adaptive design process of the structure scheme with sufficient resources, and the latter can be carried on the innovative design through the principle selection process. The algorithm flow is shown in Fig.3.

In this paper, the establishment of function-structure knowledge base in the field will support the structure to the function of the mapping process. The knowledge base contains the following stuff:

1. The direct mapping relationship between the function and the structure, including the previous definition of the atomic function and the structure to support the function; 


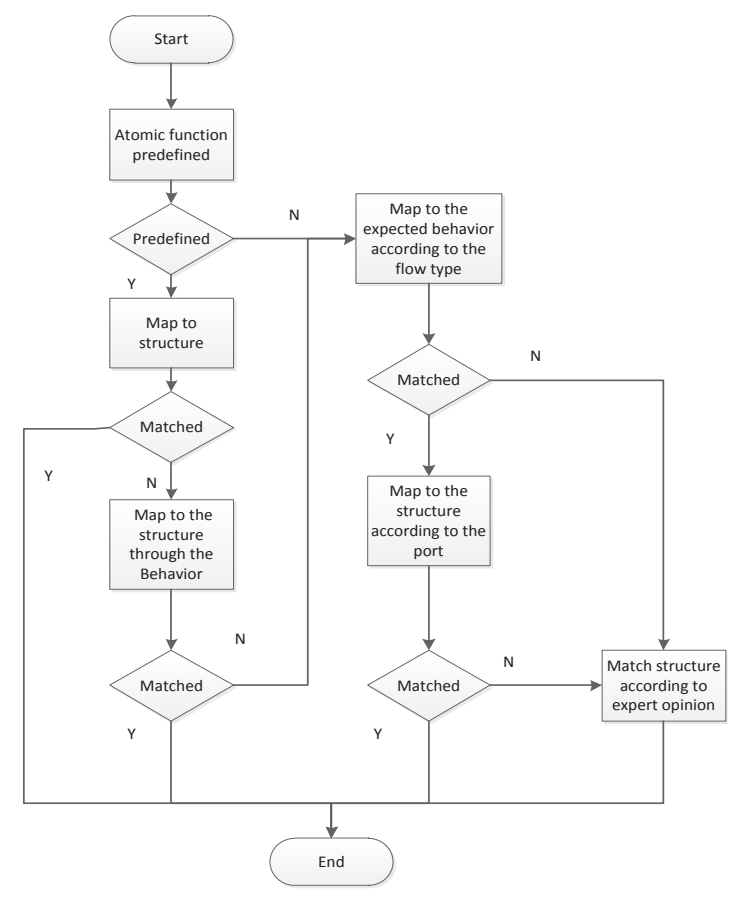

Fig. 3. The process of function-structure mapping

2. Function-behavior-structure mapping relationship, the complex atomic function, through the method of the behavior layer, then mapped to the structure.

The atomic functions decomposed in the previous step, which can be mapped to the predefined atomic functions in the original knowledge base, will be mapped to the specific structure according to the mapping relation in the function-structure knowledge base. For the absence of pre-defined atomic functions, it is necessary to analyse the behavior that supports the function, and then map to the specific structure. The mapping process is shown in the figure 3 .

After the function-structure mapping is completed, the mapping results need to be evaluated [8]. The function-structure mapping matrix is established to analyze the relationship between functions and structures. The axioms of axiomatic design are applied to determine whether the matrix is coupled. If the coupling matrix, it is necessary to map the structure again to ensure the independence among functions.

\subsection{Structure reorganization based on DSM}

After determining the system infrastructure, it is necessary to carry out structure reorganization to ensure to build the system structure that has the high polymerization within the module, and low coupling between the modules. In this paper, the following two methods are proposed to determine the process to complete the system reorganization.

1 , according to the function hierarchical structure of the reverse reorganization, structure modules are built by bottom up. The hierarchical structure of the functions decomposed by the independent function, so the functions are independent of each other and the coupling is low. The direct reverse reorganization can get a reasonable structure model
2, according to the design structure matrix (DSM). DSM is a powerful tool for product development, project planning, system engineering, organizational design, and so on. Among them, Product Architecture DSM (P-DSM) applies clustering algorithm to reduce the coupling between modules. In this paper, the resulting structures are clustered. A collection of substructures form a module to reduce the coupling between modules, improve the independence of the structure.

After the hierarchical module structure is formed, the system constraints need to be decomposed according to the system structure until the bottom layer. The design of the structure is accomplished.

\section{Expression using SysML}

Through the system requirements and the relationship between requirements that are obtained from the first step, the SysML requirements model is established. The relationship between the requirements is expressed by the requirement diagram. The use case diagram is used to define the stakeholders, Identify the system boundaries and determine the use case description of the total system function.

The function model will be expressed in the form of the activity diagram to determine the logical relationship (e.g. serial, parallel, input and output) between the different functions. At the same time, the hierarchy relationship between the activities will be expressed by the block definition diagram.

After the structure reorganization is completed, the hierarchical relationship between the structure modules is described by the package diagram. The relationship between the structure and the constraint is described by using the module definition chart and the internal module diagram. Finally, the structure is allocated to the activity diagram to build the white-box description of the functions.

\section{Implementation and case study}

The approach presented in this article is based on the release of No Magic's Cameo Systems Modeler 18.5 plug-in. By using the aircraft air conditioning system as an example, the analysis process is verified. The system interface is shown in Fig.4.

\begin{tabular}{|c|c|c|c|}
\hline Collaborate & Window Help & Design Analysis & \\
\hline - 暂。 & Create Diagr & System requirements & D \\
\hline 品 revised & 8. revised & Function decomposition & 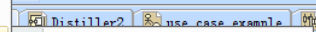 \\
\hline$\theta$ * & 㚙 - & Function-Structure Mapping ' & Mapping to Structure \\
\hline 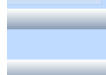 & bdd [Packas & \begin{tabular}{|l|l} 
& Structure reorganization \\
& Direct reorganization \\
DSM reorganization
\end{tabular} & $\begin{array}{l}\text { Mapping to behavior } \\
\text { behavior Mapping to Structure } \\
\text { Add to the knowledge base }\end{array}$ \\
\hline
\end{tabular}

Fig. 4. Illustration of the plug-in menu

First of all, the requirements of air conditioning system are driven from the system requirements [11]. Therefore, we don't need to get those from the users. The requirements are shown in the Fig.5. After analysis, temperature control requirements are functional requirements, and the use case diagram is established. 


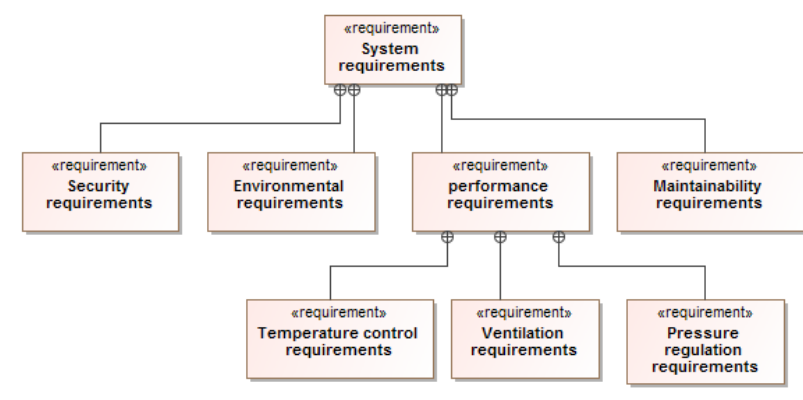

Fig. 5. The system requirement

The temperature control function is set to the total function, and decomposed. First, according to the flow type changes, it's divided into temperature changes, trim and control and warning. And then, based on the principle of decomposition, the cooling function is divided into heat exchange, heat energy into mechanical energy and so on. Therefore, it is decomposed into heat exchange and working outside. In the cooling process, the air vapor is condensed into liquid, so the moisture need to be discharged.

After the function decomposition is completed, the atomic function is mapped to the predefined structure. By matching with ports, it can be found that the drive turbine can be matched to the turbine structure. The heat exchange can be matched to the heat exchanger. Drain can be matched to the drain valve. Then the strunctures are allocated to the activities. The results need to be verified by independent axiom.

After the system structure reorganization, you can get a complete system hierarchy. The system is shown in Fig.6.

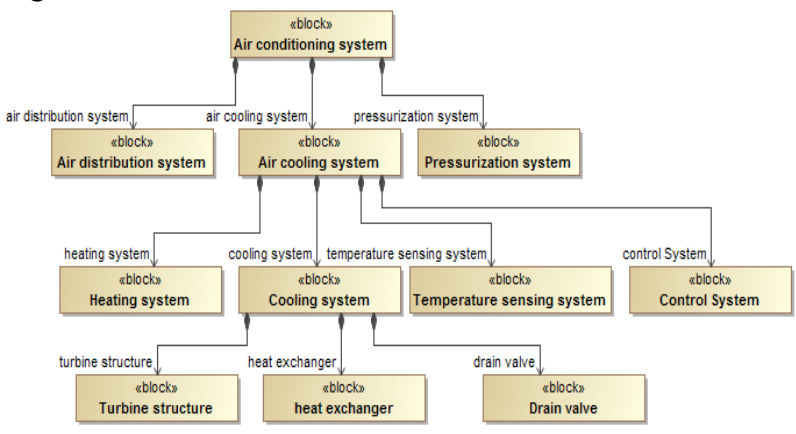

Fig. 6. The air conditioning System

\section{Conclusion and future work}

In this paper, the whole process of MBSE design analysis is established by integrating modern design method, which solves the problem that MBSE method lacks the support of scientific design theory, in order to provide designers with a complete analysis method and analysis process. Furthermore, this method makes the description of the analysis results with combining the characteristics of SysML, in order to establish a complete SysML model, and greatly improve the design efficiency and accuracy.
However, there are still limitations for this work. Analysis and mapping can only be done through a simple matching process, without the application of intelligent algorithms to provide support for the designer. The method relies on pre-defined atomic functions and structures, and lacks more creativity. The ability to support the model to automatically generate is poor. These factors will be considered and refined in the future work.

\section{Acknowledgements}

This work has been supported by Project of the National Key Technology Support Program, China (No.2015BAF17B01).

\section{References}

1. J. Holt, S. Perry, SysML for Systems Engineering (IET, 2008)

2. Haoqi W. Zhang X, System Engineering Method Based on Axiomatic Design and MBSE Modeling Framework, ASME, V01BT02A038 (2016)

3. J. A. Estefan. Survey of Model-Based Systems Engineering (MBSE) Methodologies (2008)

4. Object Management Group. Systems Modeling Language specification. (2009)

5. J. S. Gero. Design Prototypes: A Knowledge Representation Schema for Design. Ai Magazine, 11(4):26-361990 (1990)

6. Lin Y, Yusheng L, A hybrid approach for the automation of functional decomposition in conceptual design, J ENG DESIGN, 27:4-6, 333-360 (2016)

7. B. Giacomo, F. Cesare, B. Roberto, A model-based design methodology for the development of mechatronic systems, MECHATRONICS, 24, 833-843 (2014)

8. S Centre K. The Situated Function-Behaviour-Structure Framework, DESIGN STUD, 25(4), 373-391 (2001)

9. P. S. Nam., Axiomatic Design - Advances and Applications (China Machine Press, 2004)

10. S. D. Eppinger, T. R. Browning. Design structure matrix methods and applications (MIT press, 2012).

11. Xiaojin X, Shiquan L, Design Analysis of Civil Aircraft Air Conditioning System, CSTI, 16, 188 (2014)

12. MagicDraw Open API User Guide 2017. No Magic, Inc. 